\title{
An Improved Steady-State Model of an Interline Power Flow Controller for the Multi-Transmission System
}

\author{
Suresh Babu Daram $^{1 *}$, P.S.Venkataramu ${ }^{2}$, M.S.Nagaraj ${ }^{3}$ \\ ${ }^{1}$ Assistant Professor, Dept. of Electrical \& Electronics Engineering, \\ Sree Vidyanikethan Engineering College, Tirupati \& \\ Research Scholar, VTU, Belgaum \\ ${ }^{2}$ Director IQAC, REVA University, Bangalore \\ ${ }^{3}$ Professor \& Head, Dept. of Electrical \& Electronics Engineering \\ Bapuji Institute of Engineering \& Technology, Davangere \\ ${ }^{* 1}$ Corresponding author: sureshbabudaram@gmail.com, \\ ${ }^{2}$ venkataramu_ps@yahoo.com, ${ }^{3}$ msndvg\&gmail.com
}

\begin{abstract}
Flexible AC Transmission System (FACTS), under different conditions, are being incorporated in a power system for the improvement of active power flow along with voltage at each bus. Among the many FACTS devices, Interline Power Flow Controller (IPFC) is one of its kind, that has the capability of controlling multi-transmission systems. In this paper, an Improved Power Injection Model (IPIM) of Interline Power Flow Controller (IPFC) has been introduced. This model was included to Newton-Raphson (NR) method of load flow solution. The proposed model is tested on the standard test systems. The results of standard 5-bus system, IEEE-14 bus system is presented for the purpose of demonstration.
\end{abstract}

Keywords: FACTS, IPFC, Modeling of components, Multi-line transmission system, Power injected model.

\section{Introduction}

The overloading a transmission line is one of the major problems encountered by power engineers due to inadequate control of power flow. In the recent years, the investment in restructuring the transmission system has been declining steadily with a continuous rise in the demand. In this context, the control of the power flow in the line plays a major role. Flexible AC Transmission Systems (FACTS) controllers have been proved to be very useful in achieving power flow control without disturbing the generation scheduling or topological changes and in addition these devices will also enhance the secured operation of power systems [1-3].

Interline Power Flow Controller (IPFC) is one of the recent FACTS devices used for controlling the multi-line transmission power capability. The IPFC, in addition to the facility for independent controllable reactive (series) compensation of each individual line, provides a capability to directly transfer or exchange real power between the compensated lines. This is achieved by coupling the series connected Voltage Source Converters (VSC) in individual lines on the DC side, by connecting all the DC capacitors of individual converters in parallel. Since all the series converters are located inside the substation in close proximity, this is feasible [4]. The modeling of IPFC has been proposed in $[5,6]$ using voltage source converters. The importance of modeling FACTS devices in the deregulated power system has been given in [7]. 
The rest of the paper is organized as follows: Section 2 introduces the modeling of advanced steady-state model of IPFC. Section 3 explains the algorithm incorporating IPFC in the given power system. Section 4 deals with the case study and the results of the proposed algorithm. Finally, the conclusion is presented in section 5.

\section{Improved Steady-State Model of Interline Power Flow Controller}

In this section the basic load flow equations and the improved steady-state model of the IPFC is developed to incorporate in NR load flow solution.

\subsection{Basic Load Flow Equations}

The basic power flow equations [8] are as below

$$
S_{i}^{*}=V_{i}^{*} I_{i}
$$

Where

$$
\begin{aligned}
& S \text {-Apparent Power } \\
& V \text {-Voltage of a particular bus } \\
& I \text { - Current in the transmission line }
\end{aligned}
$$

From (1) the active and reactive power flow equations can be written as below

$$
V_{i}^{*} I_{i}=P_{i}-Q_{i}
$$

$$
P_{i}-Q_{i}=\left|V_{i}\right| \angle-\delta_{i} \sum_{j=1}^{n}\left|Y_{i j}\right|\left|V_{j}\right| \angle\left(\theta_{i j}+\delta_{i}\right)
$$

Where $V_{i}=V_{i} e^{j \delta_{i}}$-Voltage at bus $i$

$$
\begin{aligned}
V_{j} & =V_{j} e^{j \delta_{j}}-\text { Voltage at bus } j \\
Y_{i j} & =Y_{i j} e^{j \theta_{i j}}-\text { Admittance for the transmission line } i-j
\end{aligned}
$$

\subsection{Improved Steady state model of Interline Power Flow Controller}

Reference [6] has developed a power injection model using two Static Synchronous Series Condensers (SSSCs). The same mathematical formulations are used for the improved model of IPFC in this section. 


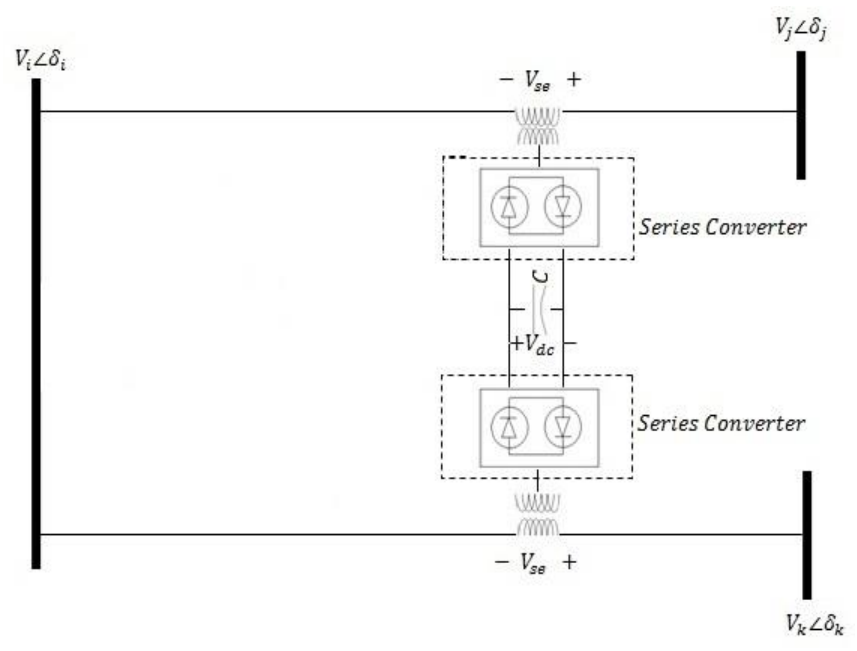

Figure 1. Conceptual hardware configuration of IPFC

Figure 1 shows the basic circuit diagram of Interline Power Flow Controller (IPFC). The power injection model has been derived from the same figure. Let the series connected voltage source converter between bus $i$ to bus $j$ is converter-I and between bus I to bus $\mathrm{k}$ is converter-II.

The equivalent circuit diagram of fig. 1 with a series connected VSC in the transmission lines $i-j \& i-k$ is shown in fig. 2 .

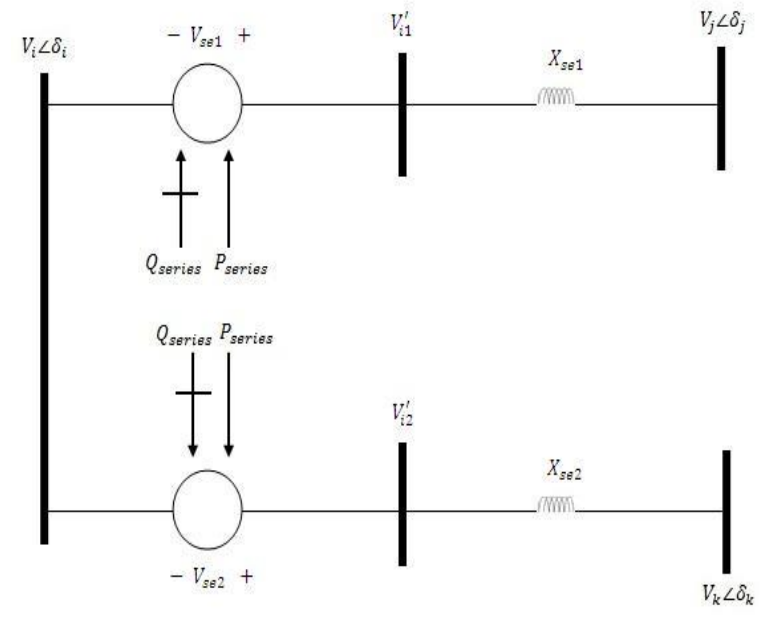

Figure 2. Equivalent Circuit of IPFC

The transformer is represented as a reactance in series with the impedance of the transmission line and a voltage source. The two series converters are controlled separately by their individual parameters.

\subsubsection{For Converter-I}

Voltage $V_{s e 1}$ of first series controller is controlled, both in magnitude and phase angle.

$V_{s e 1}=r_{1} \cdot V_{i} \cdot e^{j \gamma_{1}}$

Where $0 \leq r_{1} \leq r_{\max }$ and $-\pi \leq \gamma_{1} \leq \pi$

An imaginary voltage behind the series reactance $X_{s e l}$ is given by 
$V_{i 1}^{\prime}=V_{s e 1}+V_{i}$

\subsubsection{For Converter-II}

Voltage $V_{s e 2}$ of second series controller is controlled, both in magnitude and phase angle.

$V_{s e 2}=r_{2} \cdot V_{i} \cdot e^{j \gamma_{2}}$

Where $0 \leq r_{2} \leq r_{\max }$ and $-\pi \leq \gamma_{2} \leq \pi$

An imaginary voltage behind the series reactance $X_{s e 2}$ is given by $V_{i 2}^{\prime}=V_{s e 2}+V_{i}$

The common phasor diagram of the concerned parameters from (4)-(7) is shown in fig. 3 .

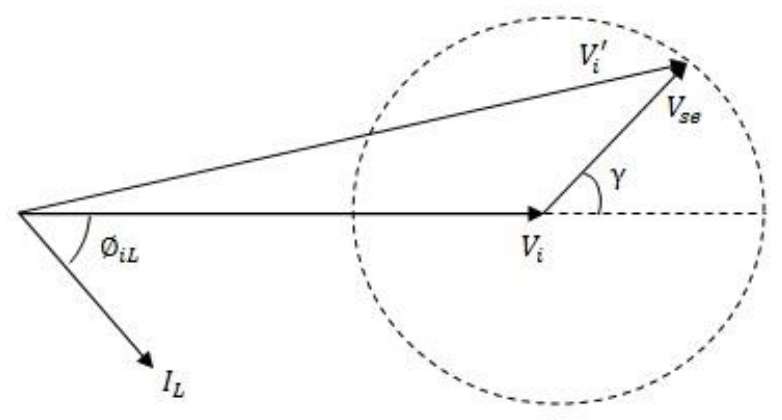

Figure 3. Phasor diagram

Voltage of bus- $i, V_{i}$ is assumed to be reference vector i.e. $V_{i}=V_{i} \angle 0$.

The power injected model can be obtained by replacing the voltage source $V_{s e}$ by a current source $I_{s e}$ in parallel with a transmission line.

Current flowing in the transmission lines can be given as

$I_{s e}=-j b_{s e} V_{s e}$

The effect of current source $I_{s e 1} \& I_{s e 2}$ and susceptance $b_{s e 1} \& b_{s e 2}$ can be modeled by injected powers at buses $i, j \& k$.

The current flowing in individual transmission lines are

$I_{s e 1}=-j b_{s e 1} V_{s e 1}$

$I_{s e 2}=-j b_{s e 2} V_{s e 2}$

The injected apparent power at bus $i$ is given as

$S_{i i n j}=V_{i}\left[-\left(I_{s e 1}+I_{s e 2}\right)^{*}\right]$

Rewriting (11), we get (12)

$S_{i i n j}=r_{1} \cdot b_{s e 1} \cdot V_{i}^{2} \cdot e^{-\left(\gamma_{1}+90\right)}+r_{2} \cdot b_{s e 2} \cdot V_{i}^{2} \cdot e^{-\left(\gamma_{2}+90\right)}$

From (12), separating injected active and reactive power, we get

$$
\begin{aligned}
& P_{i i n j}=-\left(r_{1} \cdot b_{s e 1} \cdot V_{i}^{2} \sin \gamma_{1}+r_{1} \cdot b_{s e 2} \cdot V_{i}^{2} \sin \gamma_{2}\right) \\
& Q_{i i n j}=-\left(r_{1} \cdot b_{s e 1} \cdot V_{i}^{2} \cos \gamma_{1}+r_{1} \cdot b_{s e 2} \cdot V_{i}^{2} \cos \gamma_{2}\right)
\end{aligned}
$$

The injected apparent power at bus $j$ is given as 
$S_{j i n j}=V_{j}\left(I_{s e 1}^{*}\right)$

Rewriting (15) we get (16)

$S_{i i n j}=-r_{1} \cdot b_{s e 1} \cdot V_{i} . . V_{j} \cdot e^{-\left(\delta_{i}-\delta_{j}+\gamma_{1}+90\right)}$

From (16), separating injected active and reactive power, we get

$P_{i i n j}=r_{1} \cdot b_{s e 1} \cdot V_{i} . . V_{j} \cdot \sin \left(\delta_{i}-\delta_{j}+\gamma_{1}\right)$

$Q_{i i n j}=r_{1} \cdot b_{s e 1} \cdot V_{i} . . V_{j} \cdot \cos \left(\delta_{i}-\delta_{j}+\gamma_{1}\right)$

The injected apparent power at bus $k$ is given as

$S_{k i n j}=V_{k}\left(I_{s e 2}^{*}\right)$

Rewriting (19) we get (20)

$S_{k i n j}=-r_{2} \cdot b_{s e 2} \cdot V_{i} . . V_{j} \cdot e^{-\left(\delta_{i}-\delta_{k}+\gamma_{2}+90\right)}$

From (20) separating injected active and reactive power, we get

$P_{k i n j}=r_{2} \cdot b_{s e 2} \cdot V_{i} \cdot V_{k} \cdot \sin \left(\delta_{i}-\delta_{k}+\gamma_{2}\right)$

$Q_{\text {kinj }}=r_{2} \cdot b_{s e 2} \cdot V_{i} \cdot V_{k} \cdot \cos \left(\delta_{i}-\delta_{k}+\gamma_{2}\right)$

According to the operating principle of the IPFC, active power exchange between series connected inverters via the common DC link is

$P_{\text {sum }}=\sum_{j=1, j \neq i}^{n}\left[\operatorname{Re}\left(V_{s e i j} \cdot I_{i j}\right)\right]=0$

Where

$n$ - number of buses

\subsection{Modified Jacobian equations of Newton-Raphson Power Flow solution}

By expanding (3) and neglecting all higher order terms, the set of linear expressions is shown in matrix form as below

$$
\left[\begin{array}{c}
\Delta P \\
\Delta Q
\end{array}\right]=\left[\begin{array}{cc}
H^{0} & N^{0} \\
M^{0} & L^{0}
\end{array}\right]\left[\begin{array}{c}
\Delta \delta \\
\Delta V /|V|
\end{array}\right]
$$

Where superscript ' 0 ' indicates the Jacobian elements without IPFC.

The expanded Jacobian sub-matrix elements are

$H=\frac{\partial P}{\partial \delta}, N=\frac{\partial P}{\partial V}|V|, M=\frac{\partial Q}{\partial \delta}, L=\frac{\partial Q}{\partial V}|V|$

The injected powers mentioned in section B are used to rewrite the Jacobian matrix used in Newton-Raphson power flow method.

\subsubsection{Sub-matrix H:}

The diagonal elements of $\mathrm{H}$ sub-matrix are

$\left\{\begin{array}{l}H_{i i}^{\prime}=H_{i i}^{0} \\ H_{j j}^{\prime}=H_{j j}^{0}-Q_{j i n j} \\ H_{k k}^{\prime}=H_{k k}^{0}-Q_{k i n j}\end{array}\right\}$

Off-diagonal elements of $\mathrm{H}$ sub-matrix are 


$$
\left\{\begin{array}{l}
H_{i j}^{\prime}=H_{i j}^{0} \\
H_{i k}^{\prime}=H_{i k}^{0} \\
H_{j i}^{\prime}=H_{j i}^{0}+Q_{j i n j} \\
H_{j k}^{\prime}=H_{j k}^{0} \\
H_{k i}^{\prime}=H_{k i}^{0}+Q_{k i n j} \\
H_{k j}^{\prime}=H_{k j}^{0}
\end{array}\right\}
$$

\subsubsection{Sub-matrix $N$ :}

The diagonal elements of $\mathrm{N}$ sub-matrix are

$$
\left\{\begin{array}{l}
N_{i i}^{\prime}=N_{i i}^{0}+2 P_{i i n j} \\
N_{j j}^{\prime}=N_{j j}^{0}+P_{j i n j} \\
N_{k k}^{\prime}=N_{k k}^{0}+P_{k i n j}
\end{array}\right\}
$$

Off-diagonal elements of $\mathrm{N}$ sub-matrix are

$$
\left\{\begin{array}{l}
N_{i j}^{\prime}=N_{i j}^{0} \\
N_{i k}^{\prime}=N_{i k}^{0} \\
N_{j i}^{\prime}=N_{j i}^{0}+P_{j i n j} \\
N_{j k}^{\prime}=N_{j k}^{0} \\
N_{k i}^{\prime}=N_{k i}^{0}+P_{k i n j} \\
N_{k j}^{\prime}=N_{k j}^{0}
\end{array}\right\}
$$

\subsubsection{Sub-matrix M:}

The diagonal elements of $\mathrm{M}$ sub-matrix are

$$
\left\{\begin{array}{l}
M_{i i}^{\prime}=M_{i i}^{0} \\
M_{j j}^{\prime}=M_{j j}^{0}+P_{j i n j} \\
M_{k k}^{\prime}=M_{k k}^{0}+P_{k i n j}
\end{array}\right\}
$$

Off-diagonal elements of $\mathbf{J}$ sub-matrix are

$$
\left\{\begin{array}{l}
M_{i j}^{\prime}=M_{i j}^{0} \\
M_{i k}^{\prime}=M_{i k}^{0} \\
M_{j i}^{\prime}=M_{j i}^{0}-P_{j i n j} \\
M_{j k}^{\prime}=M_{j k}^{0} \\
M_{k i}^{\prime}=M_{k i}^{0}-P_{k i n j} \\
M_{k j}^{\prime}=M_{k j}^{0}
\end{array}\right\}
$$




\subsubsection{Sub-matrix L:}

The diagonal elements of $L$ sub-matrix are

$$
\left\{\begin{array}{l}
L_{i i}^{\prime}=L_{i i}^{0}+2 Q_{i i n j} \\
L_{j j}^{\prime}=L_{j j}^{0}+Q_{j i n j} \\
L_{k k}^{\prime}=L_{k k}^{0}+Q_{k i n j}
\end{array}\right\}
$$

Off-diagonal elements of L sub-matrix are

$$
\left\{\begin{array}{l}
L_{i j}^{\prime}=L_{i j}^{0} \\
L_{i k}^{\prime}=L_{i k}^{0} \\
L_{j i}^{\prime}=L_{j i}^{0}+Q_{j i n j} \\
L_{j k}^{\prime}=L_{j k}^{0} \\
L_{k i}^{\prime}=L_{k i}^{0}+Q_{k i n j} \\
L_{k j}^{\prime}=L_{k j}^{0}
\end{array}\right\}
$$

These sub-matrices collectively form a modified Jacobian matrix with IPFC.

\section{Proposed Algorithm}

In this section the proposed algorithm is discussed. The steps in a power flow solution using the NR method after injecting IPFC in the system are as given below:

Step-1: $\quad$ Collect the Load flow data and IPFC data

Step-2: Compute $Y_{B u s}$ matrix of line data.

Step-3: $\quad$ Set iteration $\mathrm{h}=0$

Step-4: Compute modified active and reactive powers.

Step-5: Use the modified Jacobian matrix to compute the active and reactive power mismatch.

Step-6: If the power mismatch is less than the tolerable limit, it results in the output. Else go to step 7.

Step-7: Compute the change in voltage magnitude and angles using the N-R method.

Step-8: Update NR equations by fixing and varying $r_{1}, r_{2}$ and $\gamma_{1}, \gamma_{2}$ respectively and viceversa.

Step-9: $\quad$ Set $\mathrm{h}=\mathrm{h}+1$, go to step 4 .

\section{Simulation Results}

In this section, the effect of IPFC on bus voltages \& Power flows has been studied on a standard 5-bus system [9], IEEE-14 bus system [10]. The system's base MVA is 100 MVA and the tolerance limit for converging the power flow solution is considered to be $2 \times 10^{\wedge}-10$. The analysis has been carried out by varying the parameters one at a time keeping the remaining three parameters fixed. The suitable value of each parameter for the individual bus system is mentioned below separately. $\gamma_{1}, \gamma_{2}$ is varied from 0 to $2 \pi$ and $r_{1}, r_{2}$ is varied from 0.01 to 0.1 .

\subsection{Standard 5-Bus system}

For 5-bus system, there are 2 generator buses and 3 load buses and 7 transmission lines. Bus 1 is considered to be slack bus and bus- 2 as generator bus and 3, 4, 5 is load buses. Installation of IPFC near node- 4 to $3 \& 5$ as sub nodes, i.e., $4-5$ and $4-3$ are the two transmission lines, where IPFC has been incorporated in the system. The suitable values of the parameters for the power flow with 
reduced losses in the system for the existing model are $r=0.1 \& \gamma=90^{\circ}$ and for the proposed model are $r_{1}=0.1, r_{2}=0.01, \gamma_{1}=90^{\circ}, \gamma_{2}=240^{\circ}$.

The improved voltage profile of 5-bus test system is given in table-I for both IPFC models. Compared to existing model, this model has shown its superiority in terms of improved voltage profile.

Table 1. Bus Voltages for 5-Bus System

\begin{tabular}{|c|c|c|c|}
\hline \multirow{2}{*}{$\begin{array}{c}\text { Bus } \\
\text { Number }\end{array}$} & \multicolumn{3}{|c|}{ Magnitude of Voltages (p. u) } \\
\cline { 2 - 4 } & Without IPFC & With existing IPFC model [6] & With proposed IPFC model \\
\hline 1 & 1.06 & 1.06 & 1.06 \\
\hline 2 & 1.00 & 1.00 & 1.00 \\
\hline 3 & 0.987 & 0.989 & 1.031 \\
\hline 4 & 0.984 & 0.985 & 1.043 \\
\hline 5 & 0.972 & 0.970 & 1.065 \\
\hline
\end{tabular}

The active and the reactive power flows in the transmission lines are given below in Table-II \& III. It has been observed that the power flow connected to the generated bus has been reduced in the improved model. As the supply to the required load has been transferred from the lines 2-3 \& 1-3 to line 3-4. The reduction in the total system loss is $0.4 \mathrm{MW}$ and 1.2 MVAR. The active power flow has been increased and the reactive power flow has been reduced in the system. It shows proposed IPFC model has increased the transfer capability of the transmission lines when compared to the existing model.

Table 2. Active Power Flows in Transmission Line for 5-Bus System

\begin{tabular}{|c|c|c|c|c|}
\hline \multicolumn{2}{|c|}{ Line } & \multicolumn{3}{c|}{ Active Power Flow (MW) } \\
\hline From & To & Without IPFC & With existing IPFC model [6] & With proposed IPFC model \\
\hline 1 & 2 & 89.331 & 90.545 & 61.865 \\
\hline 1 & 3 & 41.791 & 40.547 & 20.134 \\
\hline 2 & 3 & 24.473 & 22.519 & 5.564 \\
\hline 2 & 4 & 27.713 & 27.922 & 26.989 \\
\hline 2 & 5 & 54.66 & 57.588 & 2.018 \\
\hline 3 & 4 & 19.386 & 16.333 & 67.833 \\
\hline 4 & 5 & 6.598 & 3.780 & 12.788 \\
\hline
\end{tabular}

Table 3. Reactive Power Flows in Transmission Line for 5-Bus System

\begin{tabular}{|c|c|c|c|c|}
\hline \multicolumn{2}{|c|}{ Line } & \multicolumn{3}{|c|}{ Active Power Flow (MW) } \\
\hline From & To & Without IPFC & With existing IPFC model [6] & With proposed IPFC model \\
\hline 1 & 2 & 73.995 & 73.639 & -120.726 \\
\hline 1 & 3 & 16.82 & 16.546 & -17.657 \\
\hline 2 & 3 & -2.518 & -2.699 & 14.023 \\
\hline 2 & 4 & -1.724 & -2.084 & 14.062 \\
\hline 2 & 5 & 5.558 & 6.424 & -55.970 \\
\hline 3 & 4 & 2.865 & 2.822 & 8.710 \\
\hline 4 & 5 & 0.518 & 0.048 & -9.208 \\
\hline
\end{tabular}




\subsection{IEEE-14 Bus System}

For IEEE-14 bus system, there are 6 generator buses and 8 load buses and 20 transmission lines. Bus 1 is considered to be slack bus, 2, 3, 6, 8 as generator buses and 4, 5, 7, 9, 10, 11, 12, 13, 14 are load buses. Installation of IPFC near bus-13 with $12 \& 14$ as sub buses, i.e. 13-12 and 13-14 are the two transmission lines, where IPFC has been incorporated in the system. The suitable values of the parameters for the power flow with reduced losses in the system for the existing model is $r=0.1 \& \gamma=120^{\circ}$ and for improved model is $r_{1}=0.15, r_{2}=0.15, \gamma_{1}=300^{\circ}, \gamma_{2}=270^{0}$.

Table 4. Bus Voltages for 14-Bus System

\begin{tabular}{|c|c|c|c|}
\hline \multirow{2}{*}{$\begin{array}{c}\text { Bus } \\
\text { Number }\end{array}$} & \multicolumn{3}{|c|}{ Magnitude of Voltages (p. u) } \\
\cline { 2 - 4 } & Without IPFC & With existing IPFC model [6] & With proposed IPFC model \\
\hline 1 & 1.0600 & 1.0600 & 1.0600 \\
\hline 2 & 1.0450 & 1.0450 & 1.0450 \\
\hline 3 & 1.0100 & 1.0100 & 1.0100 \\
\hline 4 & 1.0132 & 1.0129 & 1.0129 \\
\hline 5 & 1.0166 & 1.0165 & 1.0165 \\
\hline 6 & 1.0700 & 1.0700 & 1.0700 \\
\hline 7 & 1.0457 & 1.0448 & 1.0448 \\
\hline 8 & 1.0800 & 1.0800 & 1.0800 \\
\hline 9 & 1.0305 & 1.0289 & 1.0290 \\
\hline 10 & 1.0299 & 1.0284 & 1.0284 \\
\hline 11 & 1.0462 & 1.0453 & 1.0453 \\
\hline 12 & 1.0533 & 1.0549 & 1.0552 \\
\hline 13 & 1.0467 & 1.0482 & 1.0483 \\
\hline 14 & 1.0193 & 1.0127 & 1.0132 \\
\hline
\end{tabular}

Table 5. Active Power Flows in Transmission Line for 14-Bus System

\begin{tabular}{|c|c|c|c|c|}
\hline \multicolumn{2}{|c|}{ Line } & \multicolumn{3}{c|}{ Active Power Flow (MW) } \\
\hline From & To & Without IPFC & With existing IPFC model [6] & With proposed IPFC model \\
\hline 1 & 2 & 157.091 & 157.144 & 157.173 \\
\hline 1 & 5 & 75.519 & 75.435 & 75.450 \\
\hline 2 & 3 & 73.398 & 73.462 & 73.468 \\
\hline 2 & 4 & 55.947 & 56.041 & 56.052 \\
\hline 2 & 5 & 41.737 & 41.629 & 41.639 \\
\hline 3 & 4 & -23.135 & -23.075 & -23.070 \\
\hline 4 & 5 & -59.586 & -60.406 & -60.411 \\
\hline 4 & 7 & 27.070 & 27.690 & 27.703 \\
\hline 4 & 9 & 15.466 & 15.814 & 15.822 \\
\hline 5 & 6 & 45.897 & 44.883 & 44.902 \\
\hline 6 & 11 & 8.286 & 9.305 & 9.306 \\
\hline 6 & 12 & 8.067 & 7.196 & 7.196 \\
\hline 6 & 13 & 18.344 & 17.182 & 17.201 \\
\hline 7 & 9 & 27.070 & 27.690 & 27.703 \\
\hline 9 & 10 & 4.394 & 3.395 & 3.394 \\
\hline
\end{tabular}




\begin{tabular}{|c|c|c|c|c|}
\hline 9 & 14 & 8.642 & 10.610 & 10.631 \\
\hline 10 & 11 & -4.612 & -5.609 & -5.610 \\
\hline 12 & 13 & 1.885 & 1.031 & 1.030 \\
\hline 13 & 14 & 6.458 & 4.487 & 4.446 \\
\hline
\end{tabular}

Table 6. Reactive Power Flows in Transmission Line for 14-Bus System

\begin{tabular}{|c|c|c|c|c|}
\hline \multicolumn{2}{|c|}{ Line } & \multicolumn{3}{|c|}{ Active Power Flow (MW) } \\
\hline From & To & Without IPFC & With existing IPFC model [6] & With proposed IPFC model \\
\hline 1 & 2 & -20.453 & -20.465 & -20.472 \\
\hline 1 & 5 & 5.218 & 5.275 & 5.277 \\
\hline 2 & 3 & 3.544 & 3.538 & 3.538 \\
\hline 2 & 4 & 1.079 & 1.258 & 1.260 \\
\hline 2 & 5 & 2.849 & 2.940 & 2.942 \\
\hline 3 & 4 & 7.099 & 7.282 & 7.284 \\
\hline 4 & 5 & 11.575 & 11.280 & 11.281 \\
\hline 4 & 7 & -4.105 & -3.772 & -3.771 \\
\hline 4 & 9 & 3.453 & 3.756 & 3.757 \\
\hline 5 & 6 & 11.257 & 11.107 & 11.107 \\
\hline 6 & 11 & 8.898 & 8.880 & 8.882 \\
\hline 6 & 12 & 3.175 & 2.930 & 2.925 \\
\hline 6 & 13 & 9.979 & 9.329 & 9.326 \\
\hline 7 & 9 & 14.797 & 15.618 & 15.621 \\
\hline 9 & 10 & -0.905 & -0.845 & -0.847 \\
\hline 9 & 14 & 0.320 & 1.255 & 1.259 \\
\hline 10 & 11 & -6.721 & -6.655 & -6.657 \\
\hline 12 & 13 & 1.408 & 1.196 & 1.195 \\
\hline 13 & 14 & 5.084 & 4.280 & 4.278 \\
\hline
\end{tabular}

The bus voltages without IPFC, with existing IPFC model and with the proposed IPFC model are given in table-IV. The voltage for the buses $12 \& 13$ has been enhanced with respect to the existing IPFC model. The active and the reactive power flows in the transmission lines are given in table-V and table-VI respectively. The power flows in the transmission lines 12-13 and 13-14 has been reduced compared to the existing model. The voltage at bus 13,14 has been enhanced by $0.0001 \mathrm{pu}$ compared to the existing model and by $0.002 \mathrm{pu}$ compared to that of not included with IPFC in the system. The active power flows in the lines 12-13 \& 13-14 has reduced by $0.001 \mathrm{MW}$ compared to the existing model and by $0.8 \& 2.0 \mathrm{MW}$ respectively compared to without IPFC in the system. Which shows the controlling of voltage and power flows in the system by four parameters can be done very effectively. The reduction in the Total system loss is $0.015 \mathrm{MW}$ and 0.01 MVAR. It shows proposed IPFC model has increased the transfer capability of the transmission lines when compared to the existing model. The losses has been significantly reduced from base case 13.5929 MW to 13.578 MW with existing model and further reduced to 13.3999 MW proposed model. 


\section{Conclusion}

The improved power injection model of the multi-transmission line controller i.e., Interline Power Flow Controller with modified Jacobian matrix elements has been presented. The proposed model has been incorporated in Newton-Raphson power flow solution method to study the effects of IPFC parameters in power flow studies. Numerical results on the test system have demonstrated that the proposed IPFC model would yield fairly accurate results with regards to bus voltages and power flows. There are four controlling parameters rather than two from the basic model of IPFC. The controlling of the individual transmission line is possible with independent controlling parameter when IPFC is connected in multi transmission line. This is the advantage of the proposed power injection model. The total system loss can be controlled by choosing proper values of IPFC parameters.

\section{Acknowledgment}

The authors are grateful to the management of the Sree Vidyanikethan Engineering College, Tirupati and also to the management of Bapuji Institute of Engineering and Technology, Davangere.

\section{References}

[1] N.G. Hingorani, "Flexible AC Transmission", IEEE spectrum, (1993) April, pp 40-45.

[2] L.Gyugi, C.D.Schauder, Kalyan K.Sen, "Static synchronous series compensator: a solid-state approach to the series compenstion of transmission lines", IEEE Transaction on power delivery, Vol.12, No.1, (1997) January.

[3] L.Gyugi, C.D.Schauder, S.L.Williams, T.R.Rietman, D.R.Torgerson, A.Edris, "The Unified Power Flow Controller: A New Approach to Power Transmission Control", IEEE Transaction on Power Delivery, Vol.10, No.2, (1995)April, pp.1085-1097.

[4] Laszlo Gyugyi, "Application Characteristics of Converter-Based FACTS Controllers", Proceedings International Conference on Power Con 2000. vol.1. (2000). pp: 391 - 396

[5] X.P.Zhang, "Modelling of the interline power flow controller and the generalized unified power flow controller in Newton power flow", IEE Proc-Gener, Transm, Distrib, Vol.150, No.3, (2003) May.

[6] D. Suresh Babu, Nikhil Sahu, P S Venkataramu and M S Nagaraj, "Development of a New Model of IPFC for Power Flow in Multi-Transmission Lines," International Journal of Computer Applications 84(5):, (2013)December,pp: 33-37. Published by Foundation of Computer Science, New York, USA.

[7] K.S. Verma, S.N. Singh and H.O. Gupta, "FACTS Devices Location For Enhancement of Total Transfer Capability", IEEE Power Engineering Society, Winter Meeting, Vol.02, pp.522-527, 28-Jan-01 Feb (2001), Columbus, $\mathrm{OH}$

[8] William D .Stevenson,Jr., Elements of Power System Analysis, 4th ed, McGraw-Hill, International edition (1982), ISBN 0-07-066584-2, pp.198-199.

[9] G.W.Stagg, El-Abiad, Computer Methods in Power Systems Analysis, International Students Edition, McGraw-Hill, Kogakusha Ltd, (1968).

[10] Hadi Sadat,Power System Analysis,Tata McGraw-Hill Edition (2001).

\section{Authors}

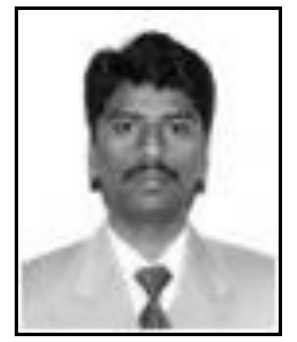

Suresh Babu Daram was born at Vijayawada (A.P), India in 1985 $\mathrm{He}$ received his B.Tech in Electrical \& Electronics engineering from JNTU in 2006 and M.Tech degree in Power Systems Engg from ANU, in 2009. He is a Research Scholar in the department of Electrical \& Electronics Engineering at the Visvesvaraya Technological University Belgaum (India)

He was Assistant Professor in the Dept. of Electrical \& Electronics at GGITM Bhopal from 2009-2015. Currently he is Asst. Prof in Dept. of Electrical \& Electronics, Sree Vidyanikethan Engineering College, Tirupati (A.P), India. He has published 20 national/ International journal / conference papers. His research interests include energy management systems, power system optimization, and 
voltage instability studies incorporating FACTS controllers and security analysis.

Mr. Suresh is a member of IEEE, AMIE (India), IAENG, CSTA, IACSIT, IRED and Student Member-ASTM.

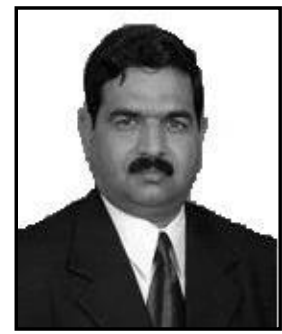

P. S. Venkataramu was born in India on June 20, 1961. He received his Graduation in Electrical Engineering from the Institute of Engineers (India), M.Tech degree in Power Systems from Mysore University (India) and Ph.D. in 2007 from Visvesvaraya Technological University, Belgaum (India).

$\mathrm{He}$ was employed as an Electrical Engineer in the Goa state Electricity Department and worked for 15 years in various capacities. He was primarily involved in carrying power system operational and planning studies for the regional grid system. He was also a visiting faculty in the Goa college of Engineering. From 1997 to 2007, he worked as a faculty in various positions at School of Electrical Sciences, Vellore Institute of Technology, Vellore, India. From 2007 to 2015 he was a Professor in Electrical Engineering Department and Principal of Gyan Ganga Institute of Technology and Management, Bhopal (India). Currently he is Director -Internal Quality at REVA University, Bangalore. He has several national and international publications. His research interest includes AI application to power system and distribution system automation.

Dr. P. S. Venkataramu is a Fellow of the institution of engineers (India), Indian society for technical education, and System society of India.

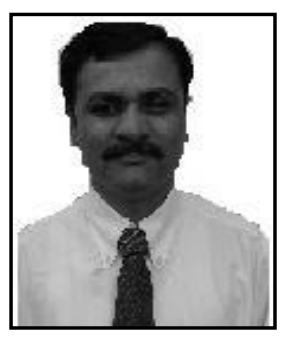

M.S.Nagaraja completed his B.E degree in Electrical \& Electronics in the year 1986 from Government BDT College of Engineering, Davangere. Master of Technology \& Doctoral degree in Power System from National Institute of Engineering, Mysore in the year $1991 \& 2007$ respectively.

He has teaching experience of 25 years in Under Graduate College. At present he is working as Professor \& Head in the department of Electrical \& Electronics Engg, BIET, Davangere. He has published 25 papers in national \& International conferences including two papers in National Journals and one International Journal. His research interest includes AI application to power system.

Dr.M.S.Nagaraja is a member of Indian Society for Technical Education and Institution of Engineers (India). 